\section{RMD Open}

Rheumatic \&

Musculoskeletal Diseases

\title{
Do patients with ankylosing spondylitis adapt to their disease? Evidence from a 'then-test' in patients treated with TNF inhibitors
}

\author{
Ivette Essers, ${ }^{1,2}$ Astrid van Tubergen, ${ }^{1,2}$ Frank Heldmann, ${ }^{3}$ Xenofon Baraliakos, ${ }^{3}$ \\ Jürgen Braun, ${ }^{3}$ Uta Kiltz, ${ }^{3}$ Annelies Boonen ${ }^{1,2}$
}

To cite: Essers I, van Tubergen A, Heldmann F, et al. Do patients with ankylosing spondylitis adapt to their disease? Evidence from a 'then-test' in patients treated with TNF inhibitors. RMD Open 2015;1:e000164. doi:10.1136/rmdopen-2015000164

- Prepublication history for this paper is available online. To view these files please visit the journal online (http://dx.doi.org/10.1136/ rmdopen-2015-000164)

Received 17 August 2015 Revised 10 October 2015 Accepted 22 October 2015

CrossMark

\begin{abstract}
${ }^{1}$ Department of Rheumatology, Maastricht University Medical Center, Maastricht, The Netherlands ${ }^{2}$ School for Public Health and Primary Care (CAPHRI), Maastricht University, Maastricht, The Netherlands ${ }^{3}$ Rheumazentrum Ruhrgebiet, Ruhr-University Bochum, Herne, Germany
\end{abstract}

Correspondence to Ivette Essers;

ivette.essers@

maastrichtuniversity.nl

\section{ABSTRACT}

Objective: To investigate whether patients with ankylosing spondylitis (AS) adapt to their disease, using the 'then-test'.

Methods: Data from patients participating in the AS Study for Evaluation of Recombinant Infliximab Therapy (ASSERT) and continuing in the European AS Infliximab Cohort (EASIC) were used. At 5 assessments in EASIC, patients were asked to rerate their global well-being before the start of infliximab in ASSERT. The patients evaluated their past situation by using a 'then-test' ('retrospective patient global'). Initial and retrospective patient global were compared using a paired $t$ test, and mixed linear models investigated whether the retrospective score of well-being was stable at all follow-up assessments in EASIC. Linear regression analysis explored whether treatment response was associated with the difference between the initial and retrospective score ('gap') while adjusting for possible confounders.

Results: 86 patients (mean age 39.8 years $(S D=10.4)$, mean disease duration 10.8 years $(\mathrm{SD}=8.5)$ ) contributed to the current analyses. At the time of starting infliximab, patients judged their global at $7.0(\mathrm{SD}=1.6)$, and with the 'then-test' at $7.2(S D=2.3)(p=0.45)$. Time elapsed did not influence the 'then-test' $(p=0.13)$. Multivariably, the gap was irrespective of treatment response, but associated with initial patient global $(p<0.01)$ and initial Bath AS Disease Activity Index ( $p=0.02$ ).

Conclusions: Patients with AS accurately judged their global well-being before starting treatment with tumour necrosis factor inhibition, even though substantial time had elapsed. The difference between initial and retrospective judgment was irrespective of treatment response. In this setting, the 'then-test' could not prove adaptation in AS.

Trial registration number: NCT01286545.

\section{INTRODUCTION}

Although never formally studied or described, rheumatologists feel that patients with ankylosing spondylitis (AS) tend to

\section{Key messages}

What is already known about this subject?

- In ankylosing spondylitis (AS), whether adaption systematically influences patients' scores on health outcomes (response shift) has never been explored.

What does this study add?

- In the setting of an open-label extension of a randomised controlled trial, patients with AS adequately recalled their global well-being before they started therapy with anti-tumour necrosis factor (TNF) (then-test).

- This retrospective judgment (then-test) was irrespective of the treatment response to anti-TNF therapy.

How might this impact on clinical practice?

- By using the 'then test', no support was found to conclude that adaptation introduced bias or response shift in self-reported health.

adjust positively to their disease. Many rheumatologists will recognise examples of patients who, during clinic visits, state, 'I'm doing well', while mobility becomes increasingly restricted and radiographic damage progresses. Along the same lines, when biologicals became available, physicians were surprised to find that some patients were reluctant to start new medications because they considered themselves capable of dealing appropriately with the disease, although rheumatologists judged them to have active disease. Classically, physicians attribute this adjustment to the slow progression of the disease, enabling patients to adapt to the new health status by developing a new internal reference frame. One previous study among 124 patients with AS confirmed that patients with AS tended to 'deny' their problems with functional activities when using 
the Minnesota Multiphasic Personality Inventory. ${ }^{1}$ Not only clinicians, who care for individual patients, but also researchers in AS, are surprised to find almost no overall progression over time at the group level for several patient-reported outcomes, such as physical function or quality of life, and wonder whether this could be a consequence of adaptation to the disease.

Adaptation can be described as a dynamic and multidimensional process of coming to terms with the implications of an acute or chronic (health) stressor and the outcomes of that process. ${ }^{2}$ Adaptation is an essential mechanism that protects humans against adverse effects of threats or chronic stressors, ${ }^{3}$ but might lead to an underestimation of the severity of a situation, which can be relevant when solutions exist. In medicine, there are three major paradigms that try to explain adjustments necessary for adaptation. ${ }^{3}$ First, the biomedical model focuses on the cause, prevention and (spontaneous or treatment supported) improvement or cure of diseases and disability. ${ }^{3}$ Second, the psychosocial model of adaptations to illness focuses on the meaning making, gaining mastery over the illness, and self-esteem. ${ }^{4}$ In this model, coping (ie, behavioural and/or cognitive responses to perceived (harmful) events) and level of self-efficacy are repeatedly implicated in the adjustment to chronic illness. The third model is the biopsychosocial model, which integrates biomedical, psychological and social aspects to understand the mechanism of adaptation. ${ }^{3}$ Being a complex and poorly understood phenomenon, it is a challenge to prove and measure adaptation, and to demonstrate its independent influence on patient-reported outcomes over time. In healthcare interventions, it is extremely relevant to prove adaptation, since the assessment of changes over time would be biased if patients use a different 'reference frame' to judge their health at two different time points. In such case, natural deterioration over time or changes in improvement due to treatment could be underestimated or overestimated.

A possible approach to reveal adaptation is the use of the 'then-test' in a situation, to assess whether there is an objective change in health. ${ }^{5}$ The 'then-test' asks a patient to recall the way the disease affected their previous health status; possibly, the gap between the initial and retrospective score of health might reflect an adaption of the previous health state. In this study, we applied the 'then-test' in patients with AS who were treated for some time with a tumour necrosis factor (TNF) inhibitor (infliximab). If patients would indeed have adapted to their disease, it would be expected that they had underestimated the true impact of AS before starting the treatment. This would lead to a better judgement of the severity of their situation at that time point, when having experienced the benefits of a treatment. Therefore, we hypothesised that patients with AS who responded to therapy would retrospectively rerate their global wellbeing as worse than initially indicated before starting on the TNF inhibitor.

\section{METHODS}

\section{Patients}

Data were retrieved from patients participating in the AS Study for the Evaluation of Recombinant Infliximab Therapy (ASSERT) and continued in the European AS Infliximab Cohort (EASIC). ASSERT included 279 adult patients with AS, according to the modified New York criteria, and these patients had high disease activity and a high spinal pain score. ASSERT started as a randomised controlled trial. During the first 24 weeks of the ASSERT trial, $201(72.0 \%)$ patients were treated with infliximab $5 \mathrm{mg} / \mathrm{kg}$ and the others were given a placebo, after which all the patients were treated with infliximab. European patients who had completed the 2-year ASSERT trial were invited to participate in EASIC, an open-label extension cohort study. The time between the last measurement of ASSERT and start of EASIC was 1.3 years. ${ }^{6}$ In total, 103 of the 216 European patients agreed to participate in EASIC, and all the patients were treated with infliximab $5 \mathrm{mg} / \mathrm{kg}$ every $6-8$ weeks. The patients were followed in EASIC for up to 96 weeks. Both study protocols were reviewed and approved by the respective institutional review board or independent medical ethics committees of each country, and all patients provided written informed consent. Details about ASSERT $^{7}$ and EASIC $^{8}$ have been previously reported.

\section{Assessments}

Baseline data were used from the ASSERT trial. In addition, baseline and follow-up data (at weeks 6, 12, 18 and 24) of EASIC were used. Information on age, sex, disease duration (time since diagnosis), disease characteristics and baseline radiographic damage, scored according to the modified Stoke AS Spine Score (mSASSS),${ }^{69}$ was retrieved from ASSERT. Disease activity was measured with the Bath AS Disease Activity Index (BASDAI),${ }^{10} \mathrm{C}$ reactive protein and the AS Disease Activity Score (ASDAS). ${ }^{11}$ Functioning was measured with the Bath AS Functional Index (BASFI) ${ }^{12}$ and spinal mobility was measured with the Bath AS Metrology Index (BASMI).$^{13}$ BASDAI $50 \%$ improvement, BASDAI $20 \%$ worsening and Assessment of SpondyloArthritis international Society 20\% (ASAS 20) response $^{14}$ were calculated based on changes in the data at baseline in ASSERT and baseline in EASIC. The patient global in the last week ('Considering all the ways your AS affects you, on average, how have you been doing in this past week?') was used to assess the effect of disease on well-being (range 0 (no effect) to 10 (very severe)), using a visual analogue scale. The patient global was assessed at baseline of ASSERT (termed 'initial patient global') and in EASIC at enrolment (termed 'current patient global'). In addition, patients were five times (at baseline and each of the 4 follow-up visits of EASIC at weeks 6, 12, 18 and 24) asked to retrospectively rerate their patient global for the time of enrolment in ASSERT (before the start of infliximab) using a 'then-test' of the patient global (termed 'retrospective patient global'). 


\section{Statistical analysis}

To describe the study population at the start of ASSERT and EASIC, means with SD for continuous data and frequencies for dichotomous data were computed.

The difference between the initial patient global of ASSERT and the first retrospective patient global in EASIC (termed 'gap') was calculated and tested for significance with a paired t test. Thereafter, the population was divided into three groups by applying a minimally clinically important difference (MCID) of 1.5 for patient global to the gap: (1) patients with a retrospective patient global at least 1.5 lower (ie, better) than the initial patient global, (2) patients with no clinically relevant difference between the retrospective patient global and the initial patient global and (3) patients with a retrospective patient global at least 1.5 higher (ie, worse) than the initial patient global. The MCID was based on the previously reported MCID of 1.5 for the Bath Ankylosing Spondylitis Patient Global Score (BAS-G). ${ }^{15}$ A Bland-Altman plot was made to visualise the gap between both assessments. The smallest detectable difference (SDD) was determined using the Bland-Altman 95\% limits of agreement method, indicating the difference in both assessments beyond the measurement error. ${ }^{16-18}$ Mixed linear models with an autoregressive correlation structure were used to investigate if the retrospective patient global (then-test) was stable over the five measurement points of EASIC.

Finally, to identify whether treatment response (defined as ASAS 20 response or BASDAI 50\% improvement) was associated with the gap, linear regression analyses were performed adjusting for several possible confounders including demographics (age, gender), and clinical (disease duration, disease activity, function, treatment) and radiographic characteristics (mSASSS) at baseline of ASSERT. A backward multivariable linear regression analysis was performed with variables with a $p$ value lower than 0.10 in the univariable analysis. Assumptions for linear regression and interactions were checked. Furthermore, we investigated if the initial, retrospective and current patient global were different between patients with and without an ASAS 20 response. Statistical analyses were performed with SPSS V.20.0.

\section{RESULTS}

A total of 103 patients were enrolled in EASIC, of whom $86(83 \%)$ retrospectively rerated their patient global before the start with infliximab. Table 1 presents the characteristics of these 86 patients at baseline in ASSERT and EASIC. At baseline in ASSERT, the mean age was 39.6 years $(\mathrm{SD}=10.6)$, and the mean disease

Table 1 Baseline characteristics of ASSERT and EASIC of the 86 patients contributing to the current study

\begin{tabular}{lll}
\hline Characteristic & Baseline ASSERT (N=86) & Baseline EASIC (N=86) \\
\hline Men & $68(79.1 \%)$ & \\
Country & $33(38.4 \%)$ & \\
$\quad$ Germany & $20(23.3 \%)$ & \\
Belgium & $21(24.4 \%)$ & \\
The Netherlands & $9(10.5 \%)$ & $43.9(10.5)$ \\
UK & $2(2.3 \%)$ & $13.0(8.0)$ \\
Finland & $1(1.2 \%)$ & $0(0 \%)$ \\
France & $39.6(10.6)$ & $86(100 \%)$ \\
Age (years) & $9.7(8.1)$ & $3.6(2.4)$ \\
Disease duration (years) & & $3.2(2.0)$ \\
Treatment & $24(27.9 \%)^{\star}$ & $0.9(1.2)$ \\
Placebo & $62(72.1 \%)^{\star}$ & $3.5(2.2)$ \\
Infliximab 5 mg/kg & $7.0(1.6)$ & $2.2(1.2)$ \\
Patient global (0-10) & $6.4(1.5)$ & - \\
BASDAI (0-10) & $2.8(2.6)$ & $68(79.1 \%)$ \\
CRP (mg/L) & $6.0(1.7)$ & $44(51.2 \%)$ \\
BASFI (0-10) & $4.2(1.9)$ & $1(1.2 \%)$ \\
BASMI (0-10) & $17.8(17.3)$ & \\
mSASSS (0-72) & - & \\
ASAS 20 response & - & \\
BASDAI 50\% improvement & - & \\
BASDAI 20\% worsening & & \\
\hline Mean (SD mean) or numb of patients) &
\end{tabular}

Mean (SD mean) or number of patients (\%).

${ }^{*}$ Results presented for the first 24 weeks of the ASSERT trial, after 24 weeks every patient received infliximab $5 \mathrm{mg} / \mathrm{kg}$ every $6-8$ weeks. ASSERT, Ankylosing Spondylitis Study for the Evaluation of Recombinant Infliximab Therapy; BASDAI, Bath Ankylosing Spondylitis Disease Activity Index; BASDAI $20 \%$ worsening, more than $20 \%$ worsening in the BASDAI between the baseline of ASSERT and baseline of EASIC; BASDAI 50\% improvement, more than 50\% improvement in the BASDAI between the baseline of ASSERT and baseline of EASIC; BASFI, Bath Ankylosing Spondylitis Functional Index; BASMI, Bath Ankylosing Metrology Index; CRP, C reactive protein; EASIC, European Ankylosing Spondylitis Infliximab Cohort; mSASSS, modified Stoke Ankylosing Spondylitis Spinal Score, ASAS 20 response, more than $20 \%$ improvement in Assessment in Ankylosing Spondylitis improvement criteria between the baseline of ASSERT and the baseline of EASIC. 
duration was 9.7 years $\quad(\mathrm{SD}=8.1)$. Disease activity (BASDAI=6.4, $\mathrm{SD}=1.5$ ) and the global impact of disease (patient global last week=7.0, $\mathrm{SD}=1.6$ ) were high. At the start of EASIC, on average 3.3 years $(\mathrm{SD}=0.5)$ after the baseline of ASSERT, BASDAI had decreased to 3.2 $(\mathrm{SD}=2.0)$, and the patient global in the last week to 3.6 $(\mathrm{SD}=2.4)$. Overall, $68(79.1 \%)$ patients fulfilled the criteria of an ASAS 20 response and 44 (51.2\%), the criteria for a BASDAI $50 \%$ improvement.

\section{Retrospective patient global versus initial patient global}

At baseline in EASIC, patients were asked to rerate their initial patient global before the start of infliximab. This retrospective patient global was, on average, slightly higher than the initial patient global (7.2 ( $\mathrm{SD}=2.3)$ versus 7.0 ( $\mathrm{SD}=1.6)$, respectively), resulting in a non-significant gap of $0.2(\mathrm{SD}=2.7, \mathrm{IQR}=-0.6$ to 1.7$)$ (table 2$)$. When ignoring the direction of this gap, the absolute difference was $1.9(\mathrm{SD}=2.0)$.

On the basis of the MCID of 1.5, 50 (58.1\%) patients had no clinically relevant gap, $13(15.1 \%)$ patients had a better (ie, lower) retrospective patient global than the initial patient global and $23(26.7 \%)$ patients had a worse (ie, higher) retrospective patient global than the initial patient global.

The distribution of the gap between the retrospective and initial patient global is presented in figure 1, and shows a trend for heteroscedasticity; that is, the gap tended to be overall negative for the lower average scores between initial and retrospective patient global, indicating patients rated the patient global retrospectively higher if the initial patient global was lower.

The SDD was 5.3, with a lower and upper limit of agreement of -5.1 and 5.5, respectively.

Table 2 shows the values of all five retrospective assessments of the initial patient global by the 'then-test'. A mixed linear model analysis with an autoregressive correlation structure showed that the gap between the

Table 2 Retrospective assessments of the patient global in comparison with initial score

\begin{tabular}{lcc}
\hline & Score & $\begin{array}{l}\text { Gap with initial } \\
\text { assessment }\end{array}$ \\
\hline Initial patient global $(0-10)$ & $7.0(1.6)$ & - \\
Retrospective patient global & $(0-10)$ & \\
Baseline EASIC & $7.2(2.3)$ & $0.2(2.7)$ \\
Week 6 EASIC & $7.6(1.9)$ & $0.6(2.4)$ \\
Week 12 EASIC & $7.2(2.3)$ & $0.2(2.7)$ \\
Week 18 EASIC & $7.2(2.6)$ & $0.2(2.9)$ \\
Week 24 EASIC & $7.7(2.6)$ & $0.7(2.6)$ \\
\hline
\end{tabular}

Mean (SD mean).

The gap between the retrospective and initial assessment of the patient global was calculated with the following formula: retrospective patient global-initial patient global.

The retrospective patient global (then-test) was measured at five time points of EASIC.

EASIC, European Ankylosing Spondylitis Infliximab Cohort.

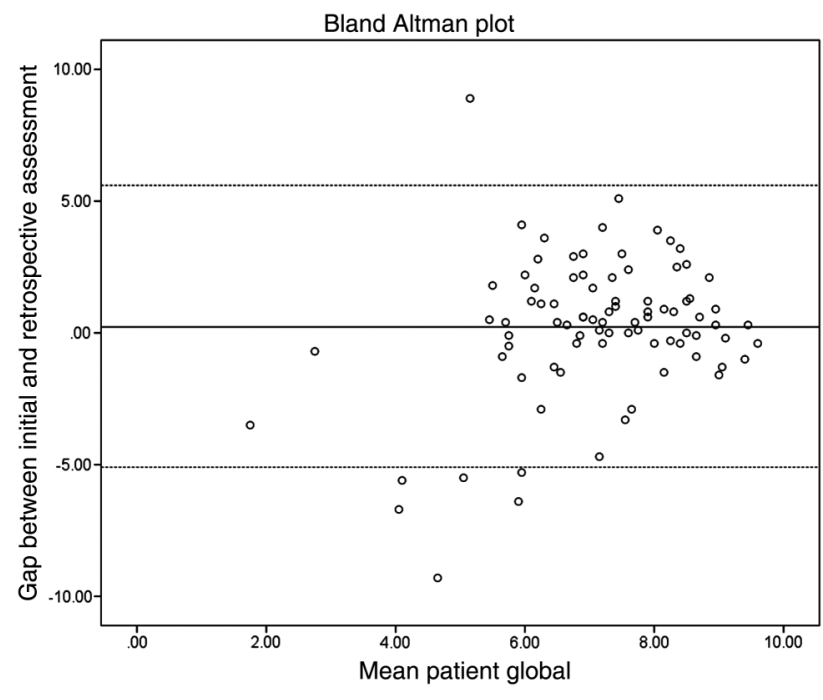

Figure 1 Bland-Altman plot of the mean of the retrospective and initial patient global plotted against the difference (gap). The mean patient global is based on the mean of the retrospective patient global and the initial patient global at baseline of Ankylosing Spondylitis Study for the Evaluation of Recombinant Infliximab Therapy (ASSERT). The gap between both assessments is based on the difference between the retrospective patient global and the initial patient global at baseline of ASSERT.

retrospective patient global, measured at five different occasions during follow-up, and initial patient global, remained constant over time (Schwarz's Bayesian Criterion=4219.997, $\mathrm{F}=1.71, \mathrm{p}=0.13$ ).

Influence of treatment response on the gap between the initial and retrospective patient global

In order to explore whether treatment response influenced the magnitude of the gap between the retrospective and initial patient global, a linear regression analysis was performed. Univariable analyses showed that the magnitude of the gap was influenced by the ASAS 20 response (between the baseline of ASSERT and baseline of EASIC, $\beta=-1.49 \mathrm{SE}=0.66, p=0.03$ ), initial patient global $(\beta=-0.09 \mathrm{SE}=0.02, p<0.01)$ and BASDAI at baseline of ASSERT $(\beta=-0.38 \mathrm{SE}=0.20, \mathrm{p}=0.05)$. However, in a multivariable linear regression analysis, only the initial patient global $(\beta=-0.12 \mathrm{SE}=0.02, \mathrm{p}<0.01)$ and BASDAI at baseline of ASSERT $(\beta=0.51 \mathrm{SE}=0.21, \mathrm{p}=0.02)$ were associated with the gap (table 3 ). Patients with a higher initial patient global showed a significantly larger negative gap, indicating that the retrospective patient global was lower (ie, better) than the initial patient global.

Figure 2 illustrates that patients with a high initial patient global (patient global $\geq 7.0$, based on the mean at baseline) had a lower retrospective patient global than the initial patient global, independently of the ASAS 20 response. Patients with a lower initial patient global had a higher retrospective patient global than the initial patient global, also independently of an ASAS 20 response. 
Table 3 Variables associated with the gap between the retrospective and initial patient global

\begin{tabular}{|c|c|c|c|c|}
\hline \multirow[b]{2}{*}{ Characteristic } & \multicolumn{2}{|l|}{ Univariable } & \multicolumn{2}{|l|}{ Multivariable } \\
\hline & $\beta$ (SE) & p Value & $\beta$ (SE) & p Value \\
\hline Male gender & $-0.63(0.72)$ & 0.38 & & \\
\hline Age (years) & $0.00(0.03)$ & 0.98 & & \\
\hline Disease duration (years) & $-0.01(0.04)$ & 0.71 & & \\
\hline Initial patient global & $-0.91(0.15)$ & $<0.01$ & $-0.12(0.02)$ & $<0.01$ \\
\hline BASDAI & $-0.38(0.20)$ & 0.05 & $0.51(0.21)$ & 0.02 \\
\hline BASDAI change & $0.19(0.15)$ & 0.19 & & \\
\hline BASDAI 50\% improvement* & $0.23(0.61)$ & 0.70 & & \\
\hline ASAS 20 response* & $-1.49(0.66)$ & 0.03 & & \\
\hline BASFI & $-0.06(0.17)$ & 0.73 & & \\
\hline BASFI change* & $0.24(0.15)$ & 0.12 & & \\
\hline BASMI & $-0.01(0.15)$ & 0.93 & & \\
\hline BASMI change* & $0.14(0.19)$ & 0.49 & & \\
\hline CRP (mg/L) & $-0.20(0.11)$ & 0.09 & & \\
\hline mSASSS & $0.06(0.18)$ & 0.75 & & \\
\hline Treatment with infliximab† & $0.51(0.66)$ & 0.44 & & \\
\hline \multicolumn{5}{|c|}{$\begin{array}{l}\text { Analysis performed with linear regression analysis, statistically significant when } p \leq 0.05 \text { (bold). } \\
\text { The gap was calculated as the retrospective patient global minus the initial patient global. } \\
\text { Baseline values of ASSERT were used unless otherwise stated. } \\
{ }^{*} \text { Change scores were calculated between the baselines of ASSERT and EASIC. } \\
\text { †Treatment with infliximab compared with placebo for the first } 24 \text { weeks. After } 24 \text { weeks all patients were treated with infliximab } 5 \text { mg/kg every } \\
6-8 \text { weeks. } \\
\text { ASAS } 20 \text { response, more than } 20 \% \text { improvement in Assessment in Ankylosing Spondylitis improvement criteria between the baseline of } \\
\text { ASSERT and the baseline of EASIC; ASSERT, Ankylosing Spondylitis Study for the Evaluation of Recombinant Infliximab Therapy; BASDAI, } \\
\text { Bath Ankylosing Spondylitis Disease Activity Index; BASDAI } 50 \% \text { improvement, more than } 50 \% \text { change in the BASDAI between the baseline } \\
\text { of ASSERT and baseline of EASIC; BASFI, Bath Ankylosing Spondylitis Functional Index; BASMI, Bath Ankylosing Metrology Index; CRP, C } \\
\text { reactive protein; mSASSS, modified Stoke Ankylosing Spondylitis Spinal Score; EASIC, European Ankylosing Spondylitis Infliximab Cohort. }\end{array}$} \\
\hline
\end{tabular}

\section{DISCUSSION}

This study showed that patients with AS who were treated with TNF inhibitors could, on average, accurately rerate their well-being before the start of treatment. As such, the current findings could not confirm our hypothesis that the 'then-test' is able to confirm adaptation in patients with AS. On this line, we found insufficient evidence that a treatment effect would be underestimated in clinical studies when response shift was not taken into account.

In the literature, the 'then-test' is used as one of the approaches to assess adaptation, as it provides empirical evidence to support either the theory of response shift ${ }^{19}$ or the implicit theory of change. ${ }^{20}$ Response shift assumes that more information is available in the 'new' situation, ${ }^{5} 13$ and that the difference between the retrospective and initial assessment will be greater when the change in health is greater, and the previous (adapted) health state might be rerated either as worse or better. ${ }^{5}$ The implicit theory of change, however, states that patients will rerate the previous health state based on their current situation, and extrapolate back, taking into account the knowledge on whether or not they 'should' have changed. ${ }^{5}$ This theory assumes that patients, being aware of receiving the best available treatment, would estimate their initial health as worse. In our study, the mean gap between the retrospective and initial patient global was 0.2 , which was neither statistically significant nor clinically relevant. ${ }^{15}$ Moreover, this gap did not represent a systematic difference, as some patients judged their previous health as similar, but also, a small proportion judged it as either better or worse. Furthermore, the gap between the retrospective and initial patient global was not associated with treatment response. Therefore, we could not demonstrate support for the response shift nor could we do so for the implicit theory of change. Importantly, we found a relation between the gap and the initial patient global, and, to a lesser extent, to the disease activity at the baseline of ASSERT. Patients who scored high on the initial patient global rerated the retrospective patient global lower and vice versa (figure 2). As such, on the individual level, the difference between the initial and retrospective patient global could be quite large. Taking into account the large within-patient variation in initial and retrospective patient global, the group seems to regress 'backwards' to a similar mean.

Although we could not prove adaptation in AS based on the 'then-test', two previously conducted studies in musculoskeletal diseases clearly demonstrated it. A study in 203 patients with chronic back pain showed that patients rerated their original quality of life to a significantly worse degree than initially reported, after inpatient rehabilitation. ${ }^{21}$ In this study, the response shift as well as the implicit theory of change could explain the difference in quality of life, as all patients improved and wanted to have faith that the treatment programme was successful. ${ }^{21}$ In contrast, another study on 197 patients with rheumatoid arthritis showed that all patients who started on TNF $\alpha$-blocking agents, both 
Figure 2 Initial, retrospective and current global well-being according to the ASAS 20 response. (A) all patients; (B) patients with an initial patient $\geq 7.0$ and $(C)$ patients with an initial patient global $<7.0$. ASAS 20 response, more than $20 \%$ improvement in Assessment of SpondyloArthritis international Society improvement criteria between the baseline of Ankylosing Spondylitis Study for the Evaluation of Recombinant Infliximab Therapy and the baseline of European Ankylosing Spondylitis Infliximab Cohort.
A

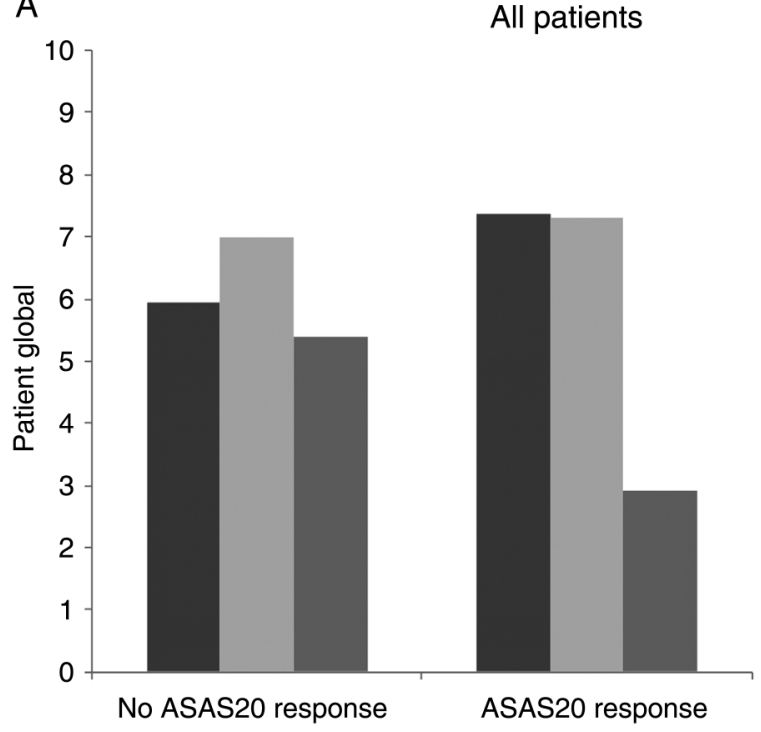

- Initial patient global

Retrospective patient global

- Current patient global
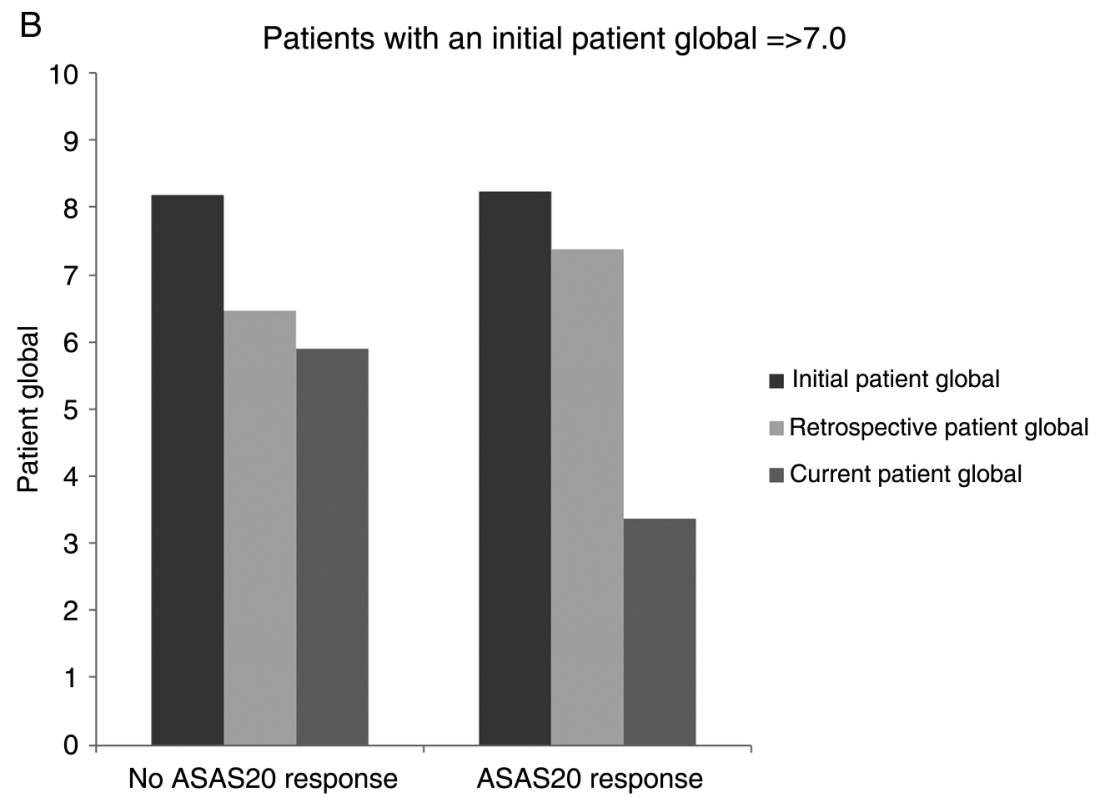

C

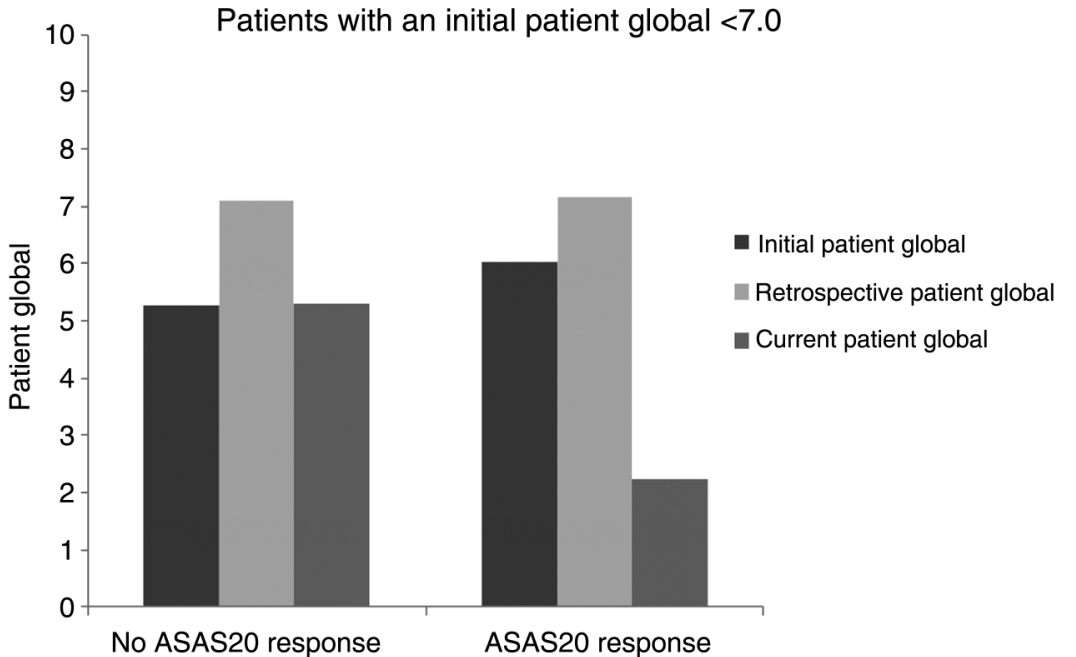


responders and non-responders, rerated their initial health as worse. ${ }^{22}$ The implicit theory of change, instead of response shift, could explain these results.

Some limitations of our study need to be addressed. First, the sample size is small because only 86 patients of the 275 patients from ASSERT participated in EASIC. Selection bias might have occurred. However, we considered it unlikely that only patients who adapted to their disease would have disagreed to continue in the follow-up study. Second, the 'then-test' itself might have some limitations, as it depends partly on the cognitive function of a patient. Specifically, recall bias might have occurred. $^{22} 23$ In our study, on average, 3.3 years had elapsed between the start of ASSERT and the first retrospective patient global assessment in EASIC. However, the gap between the retrospective patient global, measured at 5 different occasions during follow-up, and the initial patient global, remained constant over time, suggesting that recall bias did not importantly influence our results. Third, for a more complete analysis of response shift, ideally, we should have quantified not only the contribution of 'response' to the magnitude and direction of the gap, but also the contribution of 'worsening'. However, formal non-responder criteria do not exist. When we used a deterioration of the BASDAI of more than $20 \%$ as definition of worsening, only one patient fulfilled this definition, and, therefore, we were not able to perform this type of analysis.

Can we definitely conclude that patients with AS do not adapt to their disease? Actually, we can only conclude that patients accurately rerate their previous wellbeing and that there is no bias in the prospective changes that we observe in intervention studies. We cannot exclude, however, that patients, in their retrospective judgement of well-being, took into account the fact that they adapted. Although no 'objective' measure to assess patient global well-being exists, it is interesting to mention a cross-sectional study comparing experienced functional difficulties according to patients with AS with observed functional difficulties of these patients according to physicians. ${ }^{4}$ This study found that the discordance was negligible between patients and physicians, ${ }^{4}$ indicating that patients with AS report their own functional disability adequately without overestimation or underestimation. However, how these functional limitations influenced their self-reported well-being is not assessed in this study. Therefore, further research on the issue of adaptation is still of interest and could focus on life course questionnaires or interviews to assess the process of adaptation, and should relate adaptation not only to well-being but also to behaviour, including underuse or overuse of healthcare services.

In conclusion, we found insufficient evidence to presume that a treatment effect in trials is biased when response shift is not taken into account. In this study, we showed that patients with AS were able to adequately retrospectively judge their well-being, even if substantial time elapsed between the start of the treatment and this retrospective assessment. In this setting, the 'then-test' could not prove adaptation by response shift in this AS population.

Contributors All the authors were involved in drafting the manuscript or revising it critically for important intellectual content, interpretation of the data and approved the manuscript before it was submitted by the corresponding author. IE, AB and AvT had full access to all of the data in the study, and take responsibility for the integrity of the data and the accuracy of the data analysis. $\mathrm{JB}, \mathrm{UK}$, and $\mathrm{XB}$ were involved in study conception and design. FH was involved in acquisition of data. Analysis of the data IE supervised by AvT and AB.

Funding An unconditional research grant was received from MSD.

Competing interests None declared.

Patient consent Obtained.

Ethics approval METC of each country.

Provenance and peer review Not commissioned; externally peer reviewed.

Data sharing statement No additional data are available.

Open Access This is an Open Access article distributed in accordance with the Creative Commons Attribution Non Commercial (CC BY-NC 4.0) license, which permits others to distribute, remix, adapt, build upon this work noncommercially, and license their derivative works on different terms, provided the original work is properly cited and the use is non-commercial. See: http:// creativecommons.org/licenses/by-nc/4.0/

\section{REFERENCES}

1. Hidding $A$, van der Linden $S$, Boers $M$, et al. Fake good test-taking attitude in ankylosing spondylitis (abstr). Arthritis Rheum 1992; (Suppl)35:S244

2. Biesecker BB, Erby LH, Woolford S, et al. Development and validation of the Psychological Adaptation Scale (PAS): use in six studies of adaptation to a health condition or risk. Patient Educ Couns 2013;93:245-54.

3. Walker JG, Jackson HJ, Littlejohn GO. Models of adjustment to chronic illness: using the example of rheumatoid arthritis. Clin Psychol Rev 2004;24:461-88.

4. Hidding A, van Santen M, De Klerk E, et al. Comparison between self-report measures and clinical observations of functional disability in ankylosing spondylitis, rheumatoid arthritis and fibromyalgia. J Rheumatol 1994;21:818-23.

5. Norman G. Hi! How are you? Response shift, implicit theories and differing epistemologies. Qual Life Res 2003;12:239-49.

6. Ramiro S, van Tubergen A, Stolwijk C, et al. Scoring radiographic progression in ankylosing spondylitis: should we use the modified Stoke Ankylosing Spondylitis Spine Score (mSASSS) or the Radiographic Ankylosing Spondylitis Spinal Score (RASSS)? Arthritis Res Ther 2013;15:R14.

7. van der Heijde D, Dijkmans B, Geusens P, et al. Efficacy and safety of infliximab in patients with ankylosing spondylitis: results of a randomized, placebo-controlled trial (ASSERT). Arthritis Rheum 2005;52:582-91.

8. Heldmann F, Brandt J, van der Horst-Bruinsma IE, et al. The European ankylosing spondylitis infliximab cohort (EASIC): a European multicentre study of long term outcomes in patients with ankylosing spondylitis treated with infliximab. Clin Exp Rheumatol 2011;29:672-80.

9. Creemers MC, Franssen MJ, van't Hof MA, et al. Assessment of outcome in ankylosing spondylitis: an extended radiographic scoring system. Ann Rheum Dis 2005;64:127-9.

10. Garrett S, Jenkinson T, Kennedy LG, et al. A new approach to defining disease status in ankylosing spondylitis: the Bath Ankylosing Spondylitis Disease Activity Index. J Rheumatol 1994;21:2286-91.

11. Lukas C, Landewé R, Sieper J, et al. Development of an ASAS-endorsed disease activity score (ASDAS) in patients with ankylosing spondylitis. Ann Rheum Dis 2009;68:18-24.

12. Calin A, Garrett $\mathrm{S}$, Whitelock $\mathrm{H}$, et al. A new approach to defining functional ability in ankylosing spondylitis: the development of the Bath Ankylosing Spondylitis Functional Index. J Rheumatol 1994;21:2281-5.

13. Jenkinson TR, Mallorie PA, Whitelock HC, et al. Defining spinal mobility in ankylosing spondylitis (AS). The Bath AS Metrology Index. J Rheumatol 1994;21:1694-8. 
14. Sieper J, Rudwaleit M, Baraliakos X, et al. The Assessment of SpondyloArthritis international Society (ASAS) handbook: a guide to assess spondyloarthritis. Ann Rheum Dis 2009;68(Suppl 2):ii1-44.

15. Pavy S, Brophy S, Calin A. Establishment of the minimum clinically important difference for the bath ankylosing spondylitis indices: a prospective study. J Rheumatol 2005;32:80-5.

16. Auleley GR, Benbouazza K, Spoorenberg A, et al. Evaluation of the smallest detectable difference in outcome or process variables in ankylosing spondylitis. Arthritis Rheum 2002;47:582-7.

17. Bland JM, Altman DG. Statistical methods for assessing agreement between two methods of clinical measurement. Lancet 1986;1:307-10.

18. Bland JM, Altman DG. Comparing two methods of clinical measurement: a personal history. Int J Epidemiol 1995;24(Suppl 1): S7-14.
19. Schwartz CE, Sprangers MA. Methodological approaches for assessing response shift in longitudinal health-related quality-of-life research. Soc Sci Med 1999;48:1531-48.

20. Ross M. Relation of implicit theories to the construction of personal histories. Psychol Rev 1989(96):341-7.

21. Nagl M, Farin E. Response shift in quality of life assessment in patients with chronic back pain and chronic ischaemic heart disease. Disabil Rehabil 2012;34:671-80.

22. Kievit W, Hendrikx J, Stalmeier PF, et al. The relationship between change in subjective outcome and change in disease: a potential paradox. Qual Life Res 2010;19:985-94.

23. Schwartz Carolyn E, Sprangers Mirjam AG, Carey A, et al. Exploring response shift in longitudinal data. Psychology and Health 2004;19:51-69. 